\title{
Study of the seismicity temporal variation for the current seismic hazard evaluation in Val d'Agri, Italy
}

\author{
I. Baskoutas ${ }^{1}$ and A. D'Alessandro ${ }^{2}$ \\ ${ }^{1}$ Institute of Geodynamics, National Observatory of Athens, Athens, Greece \\ ${ }^{2}$ Istituto Nazionale di Geofisica e Vulcanologia, Centro Nazionale Terremoti, Rome, Italy \\ Correspondence to: A. D’Alessandro (antonino.dalessandro@ingv.it)
}

Received: 22 April 2014 - Published in Nat. Hazards Earth Syst. Sci. Discuss.: 19 June 2014

Revised: 1 October 2014 - Accepted: 2 October 2014 - Published: 2 December 2014

\begin{abstract}
This study examines the temporal variation of the seismicity in the Val d'Agri (southern Italy) and adjacent areas, for the current seismic hazard evaluation. The temporal variation of the seismicity is expressed as time series of the number of earthquakes, $b$ value of Gutenberg-Richter relationship or $b$ value of the frequency-magnitude distribution and the seismic energy released in the form of $\log E^{2 / 3}$. The analysis was performed by means of a new research tool that includes visualizing techniques, which helps the interactive exploration and the interpretation of temporal variation changes. The obtained time series show a precursory seismicity pattern, characterized by low and high probability periods, which preceded earthquakes of magnitude $M \geq 4$.0. The $75 \%$ of the examined cases were successfully correlated with a change in seismicity pattern. The average duration of the low and the high probability periods is 10.6 and 13.8 months respectively. These results indicate that the seismicity temporal variation monitoring in a given area and the recognition of the low and high probability periods can contribute to the evaluation, in regular monthly intervals, of current seismic hazard status.
\end{abstract}

\section{Introduction}

Val d'Agri is the most seismically active sector of the centralwestern Mediterranean region, having repeatedly been struck by destructive earthquakes in $1561(M=6.5), 1857(M=$ 7) and $1980(M=6.9)$. Earthquakes are characterized by predominant normal-faulting focal mechanisms, with NWoriented nodal planes, occur within a narrow seismic belt, about 20 to $40 \mathrm{~km}$ wide, centred on the axis of the Apen- nine chain (Amato et al., 1997; Valensise and Pantosti, 2001). Seismicity occurs mainly along the major seismogenic structures, such as the Irpinia Fault, which slipped during the 1980 $M=6.9$ normal-faulting event, but also at the boundary between adjacent fault segments of the active belt.

The Val d'Agri instrumental seismicity recorded in the last 30 years is low and sparse showing only two small seismic swarms recorded between 1996 April-June and 2002 February-December. The first swarm was characterized by lowmagnitude events $\left(M_{\mathrm{d}}=1.8-3.4\right)$, to the south of the basin at 2-7 km depth (Cucci et al., 2004). The second swarm consists of very few earthquakes with magnitudes ranging between $2.2 M_{\mathrm{L}}$ and $3.2 M_{\mathrm{L}}$, also clustering to the south of the Agri basin (Frepoli et al., 2005).

In this area there are many environmentally protected zones and natural parks, a sequence of shallow and deep aquifers and hydraulic network systems for storage and supply water for agricultural and civil purposes; therefore, the seismic hazard evaluation is a very important task. Usually to assess the seismic hazard, the most widespread and internationally accepted method is to estimate the peak ground motion expected in a given place by applying probabilistic or deterministic methodologies.

Nevertheless, the study of the properties of the time distribution of the seismicity can contribute also to the evaluation of the seismic danger in a given area. There are several methods that can statistically describe the properties of the time distribution of the seismicity. Among them the nonextensive statistical mechanics seem to be an interesting approach, for the description of the frequency-moment and the time and distance distributions between strong events. (Leonard et al, 2001; Parsons and Geist, 2012; Michas et 
al., 2013; Papadakis t al., 2013; Vallianatos and Sammonds, 2013; Antonopoulos et al., 2014).

The present work examines the temporal variation of the seismicity in the Agri valley (southern Italy) and adjoining region in the period 1983-2014 in order to evaluate the current status of the seismic danger and the time period between strong events, by means of FastBEE tool (Papadopoulos and Baskoutas, 2009, 2011).

This tool is suited to visualize simultaneously the temporal variation curves of common seismicity parameters like the number of earthquakes $\log N$ that occur in a certain magnitude range, the $b$ value of the frequency-magnitude distribution relation and the seismic energy released, supposing that they depict the influence of the tectonic stress, and to explore their temporal behaviour in terms of probability periods for an earthquake occurrence. In fact among these parameters, it is well established that $b$ value is related to the seismogenic procedure containing information on differences in the physics of the process that generates earthquakes (Aki, 1965; Smith, 1986, Imoto 1991; Monterroso 2003) and that depends on the stress condition and on the homogeneity of the material in the focal region being useful for seismicity interpretation (Mogi, 1967; Scholz, 1968; Wyss, 1973; Wiemer, 2002). On the other hand, the seismic energy released reveals the build-up and release of stress, depending on geotectonic characteristics. Keilis-Borok (1959) and Sadovski and Pisarenko (1983) have proposed that seismic energy in the form $\log E^{2 / 3}$, being from the physical point of view proportional to the rate of accumulation of the dynamic ruptures in the strong earthquake preparation process area, may reflect the variations of the tectonic stress in the region of the observation.

\section{Method}

FastBEE algorithm, based on the character of the seismicity parameters described previously, assumes that the temporal variation curves of these parameters represent distinct phases of a phenomenological model for the strong earthquake preparation (Popandopoulos and Baskoutas, 2011) and can be explained according to the classical models for the preparation of earthquakes and especially the phases of the consolidation model (Dobrovolski, 1991).

The characteristic onset of the temporal change, where the amount of the released seismic energy $\log E^{2 / 3}$ decreases and the simultaneous $b$ value increases as well the consequent changes of both parameters (i.e. increases of the seismic energy released and decreases of $b$ value), is characterized by two distinct and consecutive low and high probability stages before a strong earthquake occurrence (Fig. 1). This behaviour was formulated as a precursory seismicity pattern (Baskoutas et al. 2011; Baskoutas and Popandopoulos, 2014).

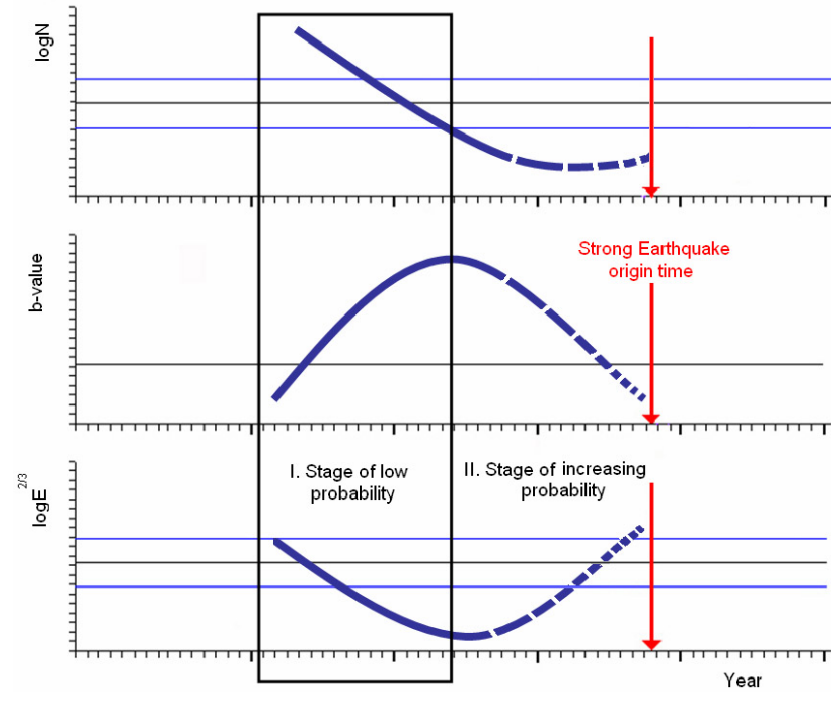

Figure 1. Characteristic FastBEE output schematic general trend of the temporal prognostic anomaly (solid blue lines) before a strong earthquake occurrence. The open rectangular parallelogram denotes the first low probability stage, since the prognostic anomaly beginning, followed by a second higher probability stage, which concludes with the strong earthquake occurrence. Vertical red arrow shows the earthquake origin time from Baskoutas and Popandopou$\operatorname{los}(2014)$.

Based on the above consideration, the methodology for the current seismic hazard evaluation consists of the following: first in the construction, by means of FastBEE tool, of the temporal variation series of a set of seismicity parameters, like the number of earthquakes $N, b$ value and the seismic energy released in the form $\log E^{2 / 3}$; and second in the correlation of the observed temporal variation series changes with the significance of the area earthquakes.

The magnitude of these events represents the lower magnitude that correlates better with the observed temporal changes and was determined by retrospective analysis of all available seismic data in the examined area. According to the FastBEE algorithm, these events depend on the seismotectonic characteristics of the area and represent, from the physical point of view, a representative response of the medium to the local tectonic stress acting in the area.

The number of earthquakes per unit time, $\log N$, is obtained by means of the following formula:

$\log N(t)=\log \left(\sum_{i=n(t-w)}^{n(t)} i\right)$,

where $i$ is the serial number of an earthquake with magnitude $M_{\mathrm{L}} \geq M_{\min } . M_{\min }$ is the completeness magnitude for sampling data, $t$ is the time (in months), $w$ is a temporary window smoothing (in months), and $n(t-w)$ is the initial number of earthquakes in the window smoothing, and $n(t)$ is the finite number of earthquakes in the window smoothing. 


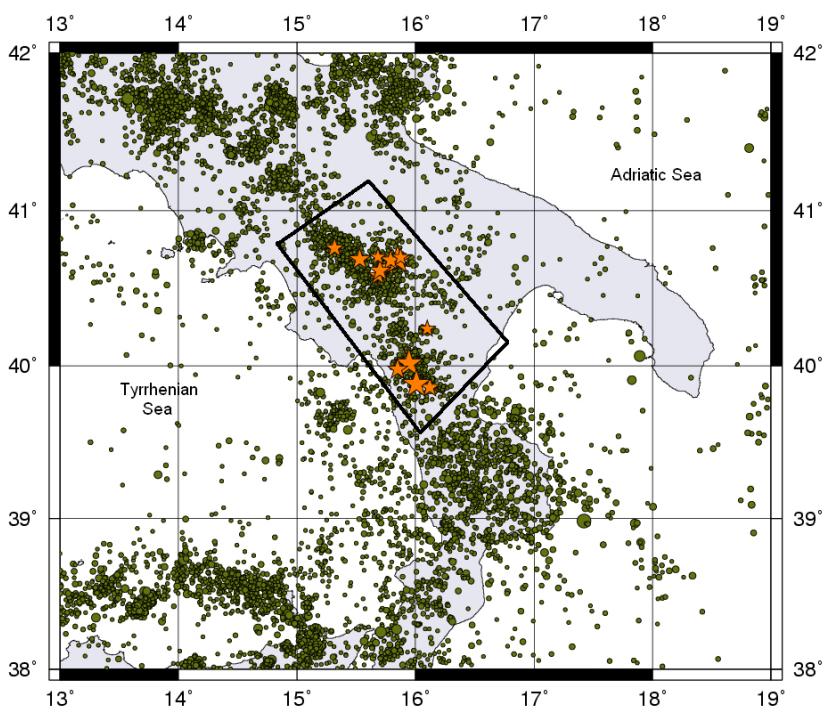

Figure 2. Seismicity map of Val d'Agri area and surrounding in the period 1983-2013 (Rectangle denotes the limits of the study area. Solid stars show the strong earthquakes, $M_{\mathrm{L}} \geq 4.0$ ).

The standard error of the calculation is given by the relation $\sigma_{\log N}=0,4343 / \sqrt{N}$.

Estimates of $b$ value are obtained using the maximum likelihood estimation method by means of the relationship proposed by Gusev (1974) as follows:

$b(t)=\log \left[1=\frac{N_{\sum}(t-w)}{\sum_{i=o}^{n} i \cdot N_{M \min +i \Delta M}(t-w)}\right] / \Delta M$,

where $t$ is the time (in months), $w$ is a temporary window smoothing (in months), $N_{\Sigma}$ is the total number of earthquakes in time interval $t-w, M_{\min }$ is the minimum completeness magnitude for earthquakes sampling data, $n=1+$ $\left(M_{\max }-M_{\min }\right) / \Delta M$ is the number of the increment (bins), $N_{M \min }+i \Delta M$ is the earthquake number in the $i$ th bin, and $\Delta M$ is a value of binned data (here $\Delta M=0.20$ ).

The standard error of the $b$ value estimates is obtained by means of the relation $\sigma_{b}(t)=b(t) / \sqrt{ } N_{\Sigma}$.

Finally $\log E^{2 / 3}$, which expresses the mean seismic energy released per unit time, is obtained using the following relation:

$\log E^{2 / 3}(t)=\log \left(\frac{1}{N(t-w)} \sum_{i=n(t-w)}^{n(t)} E_{i}^{2 / 3}\right)$,

where $E$ is the seismic energy of the earthquake, $i$ is the serial number of an earthquake with magnitude $M_{\mathrm{L}} \geq M_{\min }$. $M_{\min }$ is the minimum completeness magnitude for sampling data, $t$ is the time (in months), $w$ is a temporary window smoothing (in months), $n(t-w)$ is the initial number of earthquakes in the window smoothing, $n(t)$ is the finite number of earthquakes in the window smoothing, and $N(t-w)$

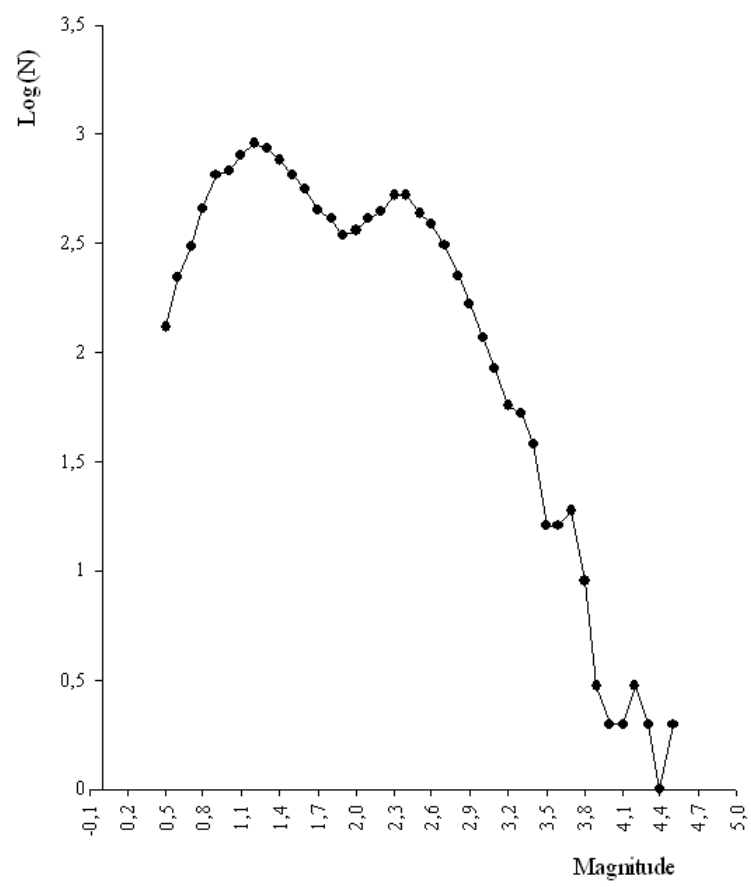

Figure 3. Non-cumulative frequency-magnitude distribution, in the period 1983-2013, denoting the changes of catalogue completeness.

is the total number of earthquakes in the temporary window smoothing. The confidence limits, for temporal variation of $\log E^{2 / 3}$, were defined as an rms (root mean square), which is calculated with the range of the examined time period.

\section{Data and analysis}

This analysis uses seismic data, in the period 1983-2013, from an area bounded by the coordinates $39.7^{\circ}-41.1^{\circ} \mathrm{N}$ and $15.1^{\circ}-16.5^{\circ} \mathrm{E}$, which includes the two main sources in the neighbourhood of seismotectonic Val d'Agri region. The data, which were taken from the earthquake catalogue of the National Institute of Geophysics and Volcanology (INGV) of Rome (Fig. 2), are complete for events with magnitudes $M \geq 2.5$ for the entire examined period. Their completeness was examined by means of the discrete frequency-magnitude distribution (Fig. 3).

Figure 4 shows, from the top to the bottom, the temporal variation of the seismic parameters $\log N, b$ value and $\log E^{2 / 3}$. Their rms scatter corresponding to a $70 \%$ confidence interval can be seen as horizontal lines on either side of the average value of the parameters $\log N$ and $\log E^{2 / 3}$. The standard error of the $b$ value, which is shown by vertical lines, refers to the monthly estimates. The temporal variation curves of the examined parameters were obtained using a moving window of $w=13$ months and with step of 1 month

The numbered arrows, perpendicular to the time axis, show the origin times of all events with $M \geq 4.0$ (Table 1); 


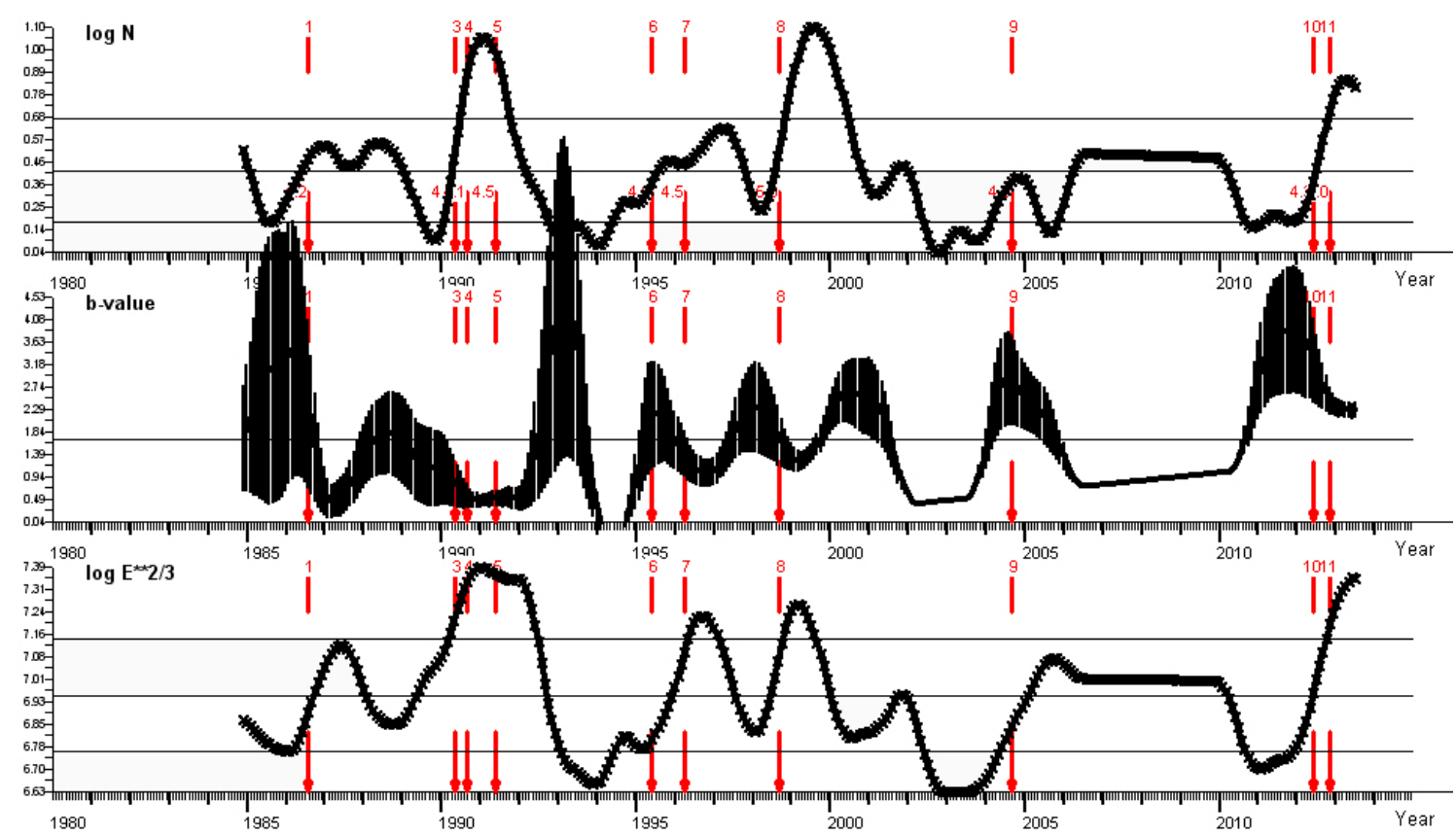

Figure 4. Temporal variation of the seismic parameters $\log N(t), b$ value and $\log E^{2 / 3}$, with their respective standard errors. Origin time and magnitude of all strong earthquakes with magnitude $M_{\mathrm{L}} \geq 4.0$ are shown as numbered arrows perpendicular to the time axis in the period 1983-2013.

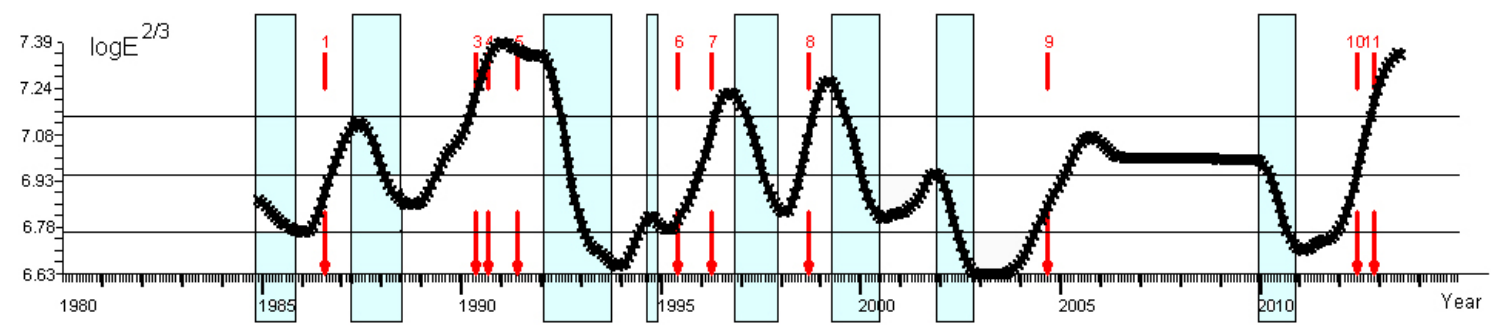

Figure 5. Temporal variation of $\log E^{2 / 3}$. Numbered arrows perpendicular to the time axis denote the origin time of all strong earthquakes, with magnitude $M_{\mathrm{L}} \geq 4.0$. Solid rectangle shows the low probability periods for an earthquake occurrence which is followed by the consecutive high probability periods.

their epicentre can be seen as solid stars in Fig. 2. This magnitude, as it was pointed out earlier, represents the lower magnitude that correlates better with the majority of the observed temporal changes, in the examined area. Due to low seismicity the magnitude range and the number of events does not permit the reliable calculation of $b$ value (Fig. 3), and hence the temporal variation of $b$ value curve (Fig. 3) will not be taken into account in the analysis.

Therefore the analysis will be based on the identification of the clear low and high probability periods of the precursory seismicity pattern (Fig. 1) on parameter $\log E^{2 / 3}$ time series only (Fig. 5). The inspection of Fig. 5 shows the existence of consecutive relative minima and maxima, over and above the mean value of the examined parameter. In the same figure, it can be seen also that all earthquakes with magnitude $M \geq 4.0$ (Table 1) are correlated with the ascending pe-
Table 1. Strong earthquakes, $M_{\mathrm{W}} \geq 5.6$, in the time period 1970-2009.

\begin{tabular}{crlllrl}
\hline s/n & yyyy mm dd & Origin time & Lat & Long & $\mathrm{h}$ & $M$ \\
\hline 1 & 1986 Jul 23 & $08: 19$ & 40.64 & 15.77 & 6 & 4.2 \\
2 & 1990 May 05 & $07: 21$ & 40.68 & 15.85 & 10 & 5.0 \\
3 & 1990 May 05 & $07: 38$ & 40.68 & 15.79 & 5 & 4.4 \\
4 & 1990 Aug 28 & $19: 02$ & 40.70 & 15.87 & 10 & 4.1 \\
5 & 1991 May 26 & $12: 26$ & 40.61 & 15.70 & 5 & 4.5 \\
6 & 1995 May 29 & $20: 44$ & 40.23 & 16.10 & 5 & 4.0 \\
7 & 1996 Apr 03 & $13: 04$ & 40.68 & 15.53 & 5 & 4.5 \\
8 & 1998 Sep 09 & $11: 27$ & 40.01 & 15.95 & 9 & 5.0 \\
9 & 2004 Sep 03 & $00: 04$ & 40.70 & 15.68 & 5 & 4.0 \\
10 & 2012 May 28 & $01: 06$ & 39.85 & 16.12 & 3 & 4.3 \\
11 & 2012 Oct 25 & $23: 05$ & 39.88 & 16.01 & 6 & 5.0 \\
\hline
\end{tabular}


Table 2. Dates of the appearance of the relative maximum and minimum of the $\log E^{2 / 3}$ time series and the low and high probability periods duration.

\begin{tabular}{cllccl}
\hline \multirow{2}{\text{s/n}}{} & Date of Max & Date of Min & $\begin{array}{c}\text { Low } \\
\text { probability } \\
\text { period } \\
\text { (group of eqs.) } \\
\text { duration }\end{array}$ & $\begin{array}{c}\text { High } \\
\text { probability } \\
\text { period } \\
\text { duration }\end{array}$ & Score \\
\hline 1 & Dec 1984 & May 1985 & 5 & 14 & Success \\
2 & May 1987 & Sep 1988 & 15 & 20 & Success \\
3 & Dec 1990 & Nov 1993 & 30 & 10 & False (till Sep 1994) \\
4 & Oct 1994 & Feb 1995 & 4 & 3 & Success \\
5 & Sep 1996 & Jan 1998 & 15 & 8 & Success \\
6 & Feb 1999 & Jul 2000 & 16 & 14 & False (till Oct 2001) \\
7 & Oct 2001 & Nov 2002 & 12 & 22 & Success \\
8 & Jan 06-Dec 07 & 46 & & Stable seismicity \\
9 & Nov 2010 & Jan 2011 & 13 & 16 & Success \\
\hline
\end{tabular}

riods of the parameter $\log E^{2 / 3}$, while the descending periods (solid rectangles in Fig. 5) show a complete absence of significant earthquakes. This behaviour indicates two clear and distinct periods of low and high stage in terms of probability.

Practically the appearance of the relative minimum, in the majority of the examined cases, signalizes the beginning of the alarm period lasting until the earthquake occurrence, unless this behaviour changes.

\section{Results}

The identification of the relative maxima and minima that represent the beginning of the two probability periods can be measured directly on these graphs or by analysing the appropriate temporal variation series.

Table 2 reports the dates of the appearance of the relative maximum that practically signalizes the beginning of the low probability period and relative minimum which signalizes the high and their respective duration, in months, which were measured using the temporal variation estimates of the parameter $\log E^{2 / 3}$.

Both qualitative and quantitative analyses show that six of the eight cases (i.e. $75 \%$ of the relative minima that appears on $\log E^{2 / 3}$ curve) were followed by one or by a group of earthquakes with magnitude $M \geq 4.0$. Instead there are two relative minima (i.e. $25 \%$ of the cases that were not followed by an earthquake); therefore, these cases can be considered as false alarms. The first of them started November 1993 and ended October 1994, and the second started July 2000 and ended October 2001.

It is interesting to point out that the temporal variation analysis of the seismic parameters reveals a period of stable seismic activity, which started at the beginning of 2006 and ended in 2009.

\section{Conclusions}

The seismicity temporal variation analysis shows significant temporal changes in all three examined seismicity parameters, using data from the Val d'Agri region in the period 1983-2012.

The form of these changes, which fluctuates around parameter mean values in the examined over 30-year period of observations, shows clear regularity corresponding to phases of a phenomenological model of earthquake preparation process. Therefore, these changes were considered to depict the response of the medium in the examined period due to the stress acting in the wider area.

Six of the eight cases of earthquakes with magnitude $M \geq$ 4.0 that occurred in this area were successfully correlated with the precursory seismicity pattern, low and high probability periods. The mean duration of the low probability period for an earthquake occurrence with magnitude $M \geq 4.0$ is about 10.6 months, while the respective high probability period is 13.8 months.

In accordance with the above findings, the evaluation of the temporal variation of seismic parameters until 2013 shows that the region is going through a period of low probability of earthquake occurrence with a magnitude above $M \geq 4.0$, unless the temporal behaviour changes. These results indicate that the continuous monitoring of the seismicity temporal variation, by means of FastBEE tool, permits the evaluation of the current seismic hazard in regular intervals, in a given area.

Acknowledgements. The authors are thankful to two anonymous reviewers for their comments that helped improve the manuscript.

Edited by: F. Vallianatos

Reviewed by: two anonymous referees 


\section{References}

Aki, K.: Maximum likelihood estimate of $\mathrm{b}$ in the formula $\log N=$ $a-b M$ and its confidence limits, Bull. Earthq. Res. Inst. Univ. Tokyo, 43, 237-239, 1965.

Amato, A., Chiarabba, C., and Selvaggi, G.: Crustal and deep seismicity in Italy (30 years after), Ann. Geofis., 40-5, 981-994, 1997.

Antonopoulos, C. G., Michas, G., Vallianatos F., and Bountis, T.: Evidence of q-exponential statistics in Greek seismicity, Physica A, 409, 71-77, 2014.

Baskoutas, I. and Popandopoulos, G.: Qualitative precursory pattern before several strong earthquakes in Greece, Res. Geophys., 4, 7-11, doi:10.4081/rg.2014.4899, 2014.

Baskoutas, I., Papadopoulos, G., and Chingtham, Pr.: Temporal variation of seismic parameters in the western part of the India-Eurasia Plate Collision Zone, Res. Geophys., doi:10.4081/rg.2011.e3, online first, 2011.

Cucci, L., Pondrelli, S., Frepoli, A., Mariucci, M. T., and Moro, M.: Local pattern of stress field and seismogenic sources in Melandro Pergola basin and in Agri valley (Southern Italy), Geophys. J. Int., 156, 575-583, 2004.

Dobrovol'skii, I. P.: Theory of Preparation of a Tectonic Earthquake, Inst. of the Phys. of the Earth. RAS, Publications, Moscow, 1991.

Frepoli, A., Cinti, F. R., Amicucci, L., Cimini, G. B., De Gori, P., and Pierdominici, S.: Pattern of seismicity in the lucanian Apennines (Southern Italy) from recording by SAPTEX temporary array, Ann. Geophys., 48, 1035-1054, 2005, http://www.ann-geophys.net/48/1035/2005/.

Gusev, A. A.: Earthquake prediction from statistical seismicity data, in Seismicity and Seismic Prediction, Upper Mantle Properties and Their Relation to the Kamchatka Volcanism, Nauka, Novosibirsk, 109-119, 1974 (in Russian).

Imoto, M.: Changes in the magnitude-frequency 6-value prior to large $(M=6.0)$ earthquakes in Japan, Tectonophysics, 193. 311-325, 1991.

Keilis-Borok, V. I.: On the estimation of the displacement in an earthquake source and of source dimensions. Annali di Geofisica 12, $205-214,1959$.

Leonard, T., Papasouliotis, O., and Main, I. G.: A Poisson model for identifying characteristic size effects in frequency data: Application to frequency-size distributions for global earthquakes, "starquakes", and fault lengths, J. Geophys. Res.-Sol. Ea., 106, 13473-13484, 2001.

Michas, G., Vallianatos, F., and Sammonds P.: Non-extensivity and long-range correlations in the earthquake activity at the West Corinth rift (Greece), Nonlinear Proc. Geophys., 20, 713-724, doi:10.5194/npg-20-713-2013, 2013.
Mogi, K.: Earthquakes and fractures, Tectonophysics, 5, 35-55, doi:10.1016/0040-1951(67)90043-1, 1967.

Monterroso Juarez, D. A.: Statistical Seismology Studies in Central America, $b$ value, seismic hazard and seismic quiescence, Comprehensive Summaries of Uppsala Dissertations from the Faculty of Science and Technology, Acta Universitalis Upsaliensis, Uppsala, 897, 27, 2003.

Papadakis, G., Vallianatos, F., and Sammonds, P.: Evidence of Nonextensive Statistical Physics behavior of the Hellenic Subduction Zone seismicity, Tectonophysics, 608, 1037-1048, doi:10.1016/j.tecto.2013.07.009, 2013.

Parsons, T., and Geist, E. L.: Were global $M \geq 8.3$ earthquake time intervals random between 1900 and 2011?, BSSA, 102, 1583-1592, doi:10.1785/0120110282, 2012.

Popandopoulos, G. A. and Baskoutas, I.: New tool for the spatiotemporal variation analysis of seismic parameters, Nat. Hazards Earth Syst. Sci., 9, 859-864, doi:10.5194/nhess-9-859-2009, 2009.

Popandopoulos, G. A. and Baskoutas, I.: Regularities in the Time Variations of the Seismic Parameters and Their Implications for Prediction of Strong Earthquakes in Greece, available online at: http://www.maik.ru/contents/physeth/physeth1011v47cont.htm, Izv Akad Nauk. Fiz Zemli, 11, 1-12, 2011.

Sadovski, M. A. and Pisarenko, V. F.: On the Dependence of the Duration of Earthquake Preparation on its Energy, Dokl. Akad. Nauk SSSR, 272, 330-333, 1983.

Scholz, C. H.: The frequency-magnitude relation of raicrofracturing in rock and its relation to earthquakes, B. Seismol. Soc. Am., 58, 399-415, 1968.

Smith, W. D.: Evidence for precursory changes in the frequency magnitude $b$ value, Geophys. J. Roy. Astr. Soc., 86, 815-838, 1986.

Valensise, G. and Pantosti, D.: The investigation of potential earthquake sources in peninsular Italy: a review, J. Seismol., 5, 287-306, 2001.

Vallianatos, F. and Sammonds P.: Evidence of non-extensive statistical physics of the lithospheric instability approaching the 2004 Sumatran-Andaman and 2011 Honshu mega-earthquakes, Tectonophysics, 590, 52-58, doi:10.1016/j.tecto.2013.01.009, 2013.

Wiemer, S. and Wyss, M.: Spatial and temporal variability of the $b$ value in seismogenic volumes: An overview, Adv. Geophys., 45, 259-302, 2002.

Wyss, M.: Towards a physical understanding of the earthquake frequency distribution, R. Astron. Soc., 31, 341-359, 1973. 